\title{
Uso de la mamografía de tamizaje en las pacientes con diagnóstico con cáncer de seno en el Hospital Universitario San Ignacio, Centro Javeriano de Oncología
}

\author{
Fernando Andrés Angarita, MD ${ }^{1}$, Sergio Andrés Acuña, MD $^{2}$, Mauricio Tawil, MD $^{3}$, \\ Álvaro J. Ruiz, MD, MSc, FACP ${ }^{4}$, Lilian Torregrosa, MD ${ }^{5}$
}

\section{Resumen}

Introducción. Actualmente el cáncer de seno ha logrado trascender en la salud de las mujeres colombianas, por ser una enfermedad con alta mortalidad. Hoy por hoy, se ofrece tamizaje de oportunidad con mamografía para mujeres mayores de 50 años, pero aún es escasa la información sobre cómo se están detectando los nuevos casos en Colombia.

Objetivo. Determinar el uso de la mamografía de tamizaje en mujeres diagnosticadas con cáncer de seno que acudieron a su primera consulta con el especialista en el Hospital Universitario San Ignacio de Bogotá, Colombia, entre el 2004 y 2007.

Materiales y métodos. Se llevó a cabo un estudio observacional descriptivo, retrospectivo, de 232 nuevos casos de cáncer de seno, de los cuales se recolectaron los siguientes datos: edad en el momento del diagnóstico, motivo de consulta, tiempo de evolución de la sintomatología, tamaño del tumor al examen físico, adenopatías, tipo histológico y estadio clínico. Para el análisis de inferencias, se aplicaron las pruebas t de student y $\times 2$ al cuadrado, según fuera apropiado, estableciendo como significativo un valor p menor de 0.05 .

Resultados. En nuestra población, sólo $18,1 \%$ de las pacientes fueron remitidas por hallazgos anormales en mamografías de tamizaje; la mayoría consultó por síntomas mamarios asociados a estadios más avanzados de la enfermedad. Aquellas que consultaron por masa, en comparación con las que lo hicieron por anormalidades en la mamografía de tamizaje, tuvieron tumores más grandes $(5,1 \mathrm{~cm}$ versus $3,3 \mathrm{~cm}$ p <0,05), con mayor compromiso linfático $(46,1 \%$ versus $14,3 \%, \mathrm{p}<0,05)$ y tendencia hacia estadios localmente avanzados (estadio III, 43,3\% versus $9,5 \%, \mathrm{p}<0.05$ ). Las mujeres mayores de 50 años tuvieron poco uso de la mamografía de tamizaje $(24,7 \%)$ y se demoraron dos veces más tiempo en consultar que las menores de 50 años $(\mathrm{p}<0,05)$.

1 Postdoctoral fellow, Toronto General Research Institute, Division of Experimental Therapeutics, University Health Network, Toronto, Canada.

2 Research associate, Peter A. Silverman Centre for International Health, Mount Sinai Hospital, Toronto, Canada.

3 Profesor titular de cirugía general, Hospital Universitario San Ignacio, Pontificia Universidad Javeriana, Bogotá, D. C., Colombia.

4 Profesor titular, Departamento de Epidemiología Clínica y Bioestadística, Facultad de Medicina, Pontificia Universidad Javeriana, Bogotá, D.C., Colombia.

5 Profesor asistente de cirugía general, Hospital Universitario San Ignacio, Pontificia Universidad Javeriana, Bogotá, D.C., Colombia. 
Conclusiones. La mayoría de los cánceres de seno se manifestaron por síntomas o signos, con una pobre detección temprana de la enfermedad. Lo anterior se refleja en un diagnóstico de cáncer en estadios avanzados. Estos hallazgos contribuyen a sustentar la urgente necesidad de desarrollar estrategias educativas efectivas, dirigidas a mujeres y profesionales de la salud, referentes al uso de la mamografía, el autoexamen y el examen clínico como herramientas de detección temprana de la enfermedad.

Palabras clave: neoplasias de la mama, mamografía, tamizaje, Colombia.

\section{Title}

Use of screening mammograms in patients diagnosed with breast cancer at Hospital Universitario San Ignacio, Centro Javeriano de Oncología

\section{Abstract}

Introduction: Breast cancer has currently become a health issue amongst Colombian women due its high mortality rate. Nowadays opportunity screening is offered with mammograms for women over 50, nonetheless little information is available on how new cases are being detected in Colombia.

Objective: Determine the use of screening mammograms in women with breast cancer who had an initial consultation with breast surgeons at Hospital Universitario San Ignacio in Bogotá, Colombia, between 2004 and 2007.

Methods: A retrospective, descriptive observational study of 232 newly diagnosed breast cancer cases was carried out in which the following data were collected: age at time of diagnosis, chief complaint, duration of symptoms, tumor size on clinical breast examination, lymph nodes, histological type, and clinical stage. For inferential analysis student's $T$ and chi square test were used. Significance was set at $\mathrm{p}<0.05$.

Results: In this population, only $18.1 \%$ of the patients were referred due to abnormal findings on screening mammograms, while the vast majority had breast symptoms as a consequence of advanced disease. Those who had lumps, in comparison to asymptomatic patients, had larger tumors $(5.1 \mathrm{~cm}$ versus $3.3 \mathrm{~cm}, \mathrm{p}<0.05)$, larger lymph node involvement ( $46.1 \%$ versus $14.3 \%$, $\mathrm{p}<0.05)$, and had a stronger tendency of locally advanced disease (stage III $43.3 \%$ versus $9.5 \%$, $\mathrm{p}<0.05$ ). Women over 50 had a low use of screening mammograms (24.7\%) and waited twice as much before having an appointment with a specialist, when compared to those less than $50(\mathrm{p}<0.05)$.

Conclusions: The majority of breast cancer cases were symptomatic, and early detection was small in this population; as a consequence, advanced disease was diagnosed in a larger proportion. These results contribute to support the growing need to develop strategies to teach women and health professionals about the benefits of mammograms, breast self exam, and clinical breast exam as tools for early detection.

Key words: breast cancer, mammogram, screening, Colombia.

\section{Introducción}

El cáncer de seno es un problema de salud pública prioritario a nivel mundial, pues constituye la primera causa de muerte por cáncer entre la población femenina y su incidencia se ha incrementado en las últimas décadas[1]. Aunque en Colombia la incidencia del cáncer de seno es menor que en los países desarrollados, se ha reportado que las mujeres provenientes de países con menores ingresos, tienen una menor supervivencia, atribuible al elevado porcentaje de casos diagnosticados en estadios tardíos de la enfermedad[2]. Lo anterior se refleja en una clara tendencia al incremento de la mortalidad en el último decenio, pese a la aparición de gran- 
des avances diagnósticos y terapéuti$\cos [1,3]$.

La magnitud actual del problema ha generado iniciativas globales que buscan detectar la enfermedad en los estadios iniciales por medio de estrategias de tamizaje y detección temprana. Esto es importante pues el diagnóstico temprano, así como el tratamiento oportuno, han demostrado mejorar la supervivencia de estas mujeres[4].

En los países desarrollados, el tamizaje organizado ha logrado reducir de manera importante las tasas de mortalidad[5-11]; este tipo de tamizaje cuenta con un centro administrativo estructurado que organiza la intervención, con controles periódicos de calidad y campañas de concientización que logran obtener diagnósticos en los estadios incipientes[12]. La implementación de este tipo de tamizaje en Colombia está limitada por los costos y la complejidad técnica[13]. Se han elaborado recomendaciones locales, publicadas por el Instituto Nacional de Cancerología que sugieren la identificación oportuna en toda mujer que presente síntomas o signos, y la detección temprana mediante el examen clínico de la mama y el tamizaje de oportunidad con mamografía en mujeres entre los 50 y los 69 años[14]. Este tipo de tamizaje deja a discreción del médico la realización de mamografías en mujeres asintomáticas y no emplea ninguna estrategia organizada de captura y seguimiento de esta población[15,16].

La reglamentación oficial respecto a la detección temprana del cáncer de seno, establecida a partir de la Resolución 412 de 2000[17], establece que la mamografía debe realizarse cada dos años, en las mujeres entre 50 y 69 años, pertenecientes al régimen contributivo y subsidiado, y contempla indicadores de cumplimiento y metas de cobertura dentro del Sistema General de Seguridad Social de Colombia.

Los desarrollos adicionales en este campo de la prevención deben ir orientados de acuerdo con un conocimiento de la población en la cual se van a emplear. Se ha descrito, por ejemplo, que en los países en vías de desarrollo el diagnóstico tardío del cáncer de seno puede estar asociado a factores culturales, sociodemográficos y algunos relacionados con el acceso y las características del sistema de sa$\operatorname{lud}[2,18]$.

De todo lo anterior surge el cuestionamiento sobre el uso de la mamografía como herramienta de tamizaje en la detección inicial del cáncer de seno en nuestra población. Para resolver este interrogante se propuso determinar cuáles habían sido el uso y el impacto de mamografía de tamizaje en las mujeres diagnosticadas con cáncer de seno que acudían a su primera con- 
sulta con el especialista en el Hospital Universitario San Ignacio de Bogotá, Colombia.

\section{Materiales y métodos}

Se realizó un estudio observacional descriptivo y retrospectivo de los nuevos casos atendidos en la Clínica de Seno y Tejidos Blandos del Centro Javeriano de Oncología entre enero de 2004 y agosto de 2007. Este centro médico, afiliado al Hospital Universitario San Ignacio, atiende, aproximadamente, 18.500 pacientes anualmente, de los cuales, 969 pacientes son atendidos en la Clínica de Seno y Tejidos Blandos.

Se revisaron todas las historias clínicas de pacientes cuyo diagnóstico hubiera sido "tumor maligno de la mama" durante el periodo de estudio. Se incluyeron las pacientes de sexo femenino con cáncer de seno que habían asistido por primera vez a la valoración con el cirujano especialista. Se excluyeron las pacientes previamente tratadas, los casos de recurrencia, aquellas con diagnóstico no establecido dentro del período de estudio, con diagnóstico de carcinomas no originarios del seno o que habían tenido su primera cita con el especialista por fuera del hospital. De las 974 historias clínicas revisadas, $232(23,8 \%)$ fueron aptas para el estudio.

Los datos a continuación se recolectaron mediante un programa de bases de datos para asistentes digitales personales que, posteriormente, lo exportaba a formato Excel, Microsoft, USA: edad en el momento del diagnóstico, motivo de consulta, tiempo de evolución de la sintomatología (si aplicaba), tamaño del tumor al examen físico, adenopatías, tipo histológico y estadio clínico.

Para el análisis estadístico descriptivo, los datos recolectados se analizaron mediante el programa Stata 10.0 (StataCorp, College Station, TX, USA), utilizando medias, medianas, desviación estándar (DE) e intervalos de confianza (IC). En cuanto al análisis de inferencias, se utilizaron las pruebas $t$ de Student y de $\times 2$, según fuera apropiado. Se determinó como significativo un valor de $\mathrm{p}$ menor de 0,05 .

\section{Resultados}

\section{Características generales de la población}

Las principales características de los 232 nuevos casos de cáncer de seno diagnosticados durante el periodo de estudio en el Centro Javeriano de Oncología, se resumen en la tabla 1.

En las 232 pacientes, la edad promedio fue de 55 años ( $\mathrm{DE}=13,5$ años; mediana=54; rango: 21 a 92 años); $63,4 \%$ de nuestros casos se presentaron en mujeres mayores de 50 años. 
Tabla 1. Características de la población $(n=232)$

\begin{tabular}{ll}
\hline Características & n (\%) \\
\hline Sexo femenino & $232(100)$ \\
Edad promedio (años) al momento del diagnóstico & 55 \\
Motivo de consulta & \\
Sintomatología (masa, telorragia, mastalgia y/o cambio en piel) & $190(81,9)$ \\
Mamografía de tamizaje anormal & $42(18,1)$ \\
Tipo histológico en la biopsia & \\
Ductal in situ & $16(6,9)$ \\
Infiltrante & $209(90,1)$ \\
Otros & $7(3,0)$ \\
Estadio clínico & \\
0 & $16(7,8)$ \\
I & $38(16,4)$ \\
IIA & $40(17,2)$ \\
IIB & $37(15,9)$ \\
IIIA & $22(9,5)$ \\
IIIB & $58(25,0)$ \\
IIIC & $4(1,3)$ \\
IV & $17(7,3)$ \\
\hline
\end{tabular}

\section{Motivos de consulta y características del tumor}

En cuanto al motivo de consulta, $190(81,9 \%)$ pacientes consultaron por sintomatología, principalmente por presencia de masa en $178(76,7 \%)$ casos. Las restantes 42 pacientes $(18,1 \%)$ fueron remitidas por anormalidades imaginológicas sin presencia de síntomas o signos clínicos, por lo que se puede suponer que se trata de casos en los que se empleó la mamografía como herramienta de tamizaje. Las pacientes que consultaron por sintomatología, en su totalidad, tenían imágenes diagnósticas de respaldo.
Al examen físico, 2,6\% de las pacientes tenían examen mamario normal, lo cual es discordante con el hecho de que $18,1 \%$ de las pacientes habían sido remitidas por anomalías imaginológicas con examen físico reportado como normal. De las pacientes remitidas por mamografía de tamizaje anormal, $85,7 \%$ presentaron hallazgos anormales al examen clínico de mama realizado por el especialista.

El tamaño promedio de los tumores en el examen físico fue de $4,2 \mathrm{~cm}$ (DE=2,2 cm; mediana $=4 \mathrm{~cm}$; rango: 1 a $10 \mathrm{~cm}$ ). En total, 89 pacientes $(38,4 \%)$ tenían adenopatías axilares palpables. 
En la tabla 2 se comparan las pacientes diagnosticadas por la presencia de síntomas con aquellas diagnosticadas mediante tamizaje de oportunidad.

En la tabla 3 se comparan los diferentes aspectos de presentación de las pacientes, según el grupo de edad en el momento del diagnóstico.

\section{Tiempo de evolución}

El tiempo promedio de evolución del cuadro clínico referido desde el inicio de la sintomatología hasta el momento en que fueron valorados en la Clínica de Seno y Tejidos Blandos fue de 9,9 meses (rango: 0,5 a 180 meses; mediana $=5,5$ meses).

\section{Discusión}

En los países desarrollados, una gran proporción de casos de cáncer de seno son detectados mediante la mamografía de tamizaje (36 a 49\%)[20, 23], lejos de la situación de nuestra población, en la cual sólo $24,5 \%$ de las pacientes fueron diagnosticadas de esta manera. El impacto sobre el pronóstico de este grupo de pacientes es importante, pues cuando los tumores son detectados

Tabla 2. Diferencias en motivos de consulta en la población

\begin{tabular}{lccc}
\hline Parámetro & $\begin{array}{c}\text { Sintomatología } \\
(\mathbf{n = 1 7 8})\end{array}$ & $\begin{array}{c}\text { Tamizaje de oportunidad } \\
(\mathbf{n = 4 2})\end{array}$ & $\begin{array}{c}\text { Valor } \\
\mathbf{P}\end{array}$ \\
\hline Edad promedio (años) & 53,9 & 59,6 & 0,0099 \\
Mayores de 50 años [n (\%) & $103(57,9)$ & $36(85,7)$ & 0,001 \\
Tiempo de evolución (meses) & 9,7 & $*$ & - \\
Tamaño de la lesión $\dagger(\mathrm{cm})$ & 5,1 & 3,3 & 0,0009 \\
Adenopatías [n (\%)] & $82(46,1)$ & $6(14,3)$ & 0,00001 \\
Tipo histológico & & & \\
Infiltrante [n (\%)] & $170(95,5)$ & $36(85,7)$ & \\
In situ n $(\%)$ & $8(4,5)$ & $6(14,5)$ & \\
Estadio clínico & & & \\
0 [n $(\%)]$ & $8(4,5)$ & $6(14,3)$ & \\
I [n $(\%)]$ & $21(11,8)$ & $15(35,7)$ & \\
II [n $(\%)]$ & $57(32,0)$ & $15(35,7)$ & \\
III [n $(\%)]$ & $77(43,3)$ & $4(9,5)$ & \\
IV [n $(\%)]$ & $15(8,4)$ & $2(4,8)$ & \\
\hline
\end{tabular}

\footnotetext{
*: no se puede calcular porque no hay tiempo de evolución de síntomas.

$\dagger$ : encontrada en el examen físico.

†: para los estadios categorizados II y III.
} 
Tabla 3. Diferencias según edad de presentación de la población

\begin{tabular}{lllc}
\hline Parámetro & $\begin{array}{c}<\mathbf{5 0} \text { años } \\
(\mathbf{n = 8 5})\end{array}$ & $\begin{array}{c}\mathbf{= 5 0} \text { años } \\
(\mathbf{n = 1 4 7})\end{array}$ & $\begin{array}{c}\text { Valor } \\
\mathbf{p}\end{array}$ \\
\hline Motivo de consulta & & & \\
Síntoma [n (\%)] & $79(92,9)$ & $111(75,5)$ & 0,001 \\
Mamografía de tamizaje [n (\%)] & $6(7,1)$ & $36(24,5)$ & \\
Tiempo de evolución (meses) & 6,5 & 12,5 & 0,04 \\
Tamaño de la lesión* (cm) & 4,8 & 4,7 & $\mathrm{NS}$ \\
Adenopatías [n (\%)] & $35(41,2)$ & $54(37,0)$ & $\mathrm{NS}$ \\
Estadio clínico & & & \\
0 [n $(\%)]$ & $2(2,4)$ & $14(9,5)$ & $\mathrm{NS}$ \\
I [n $(\%)]$ & $9(10,6)$ & $29(19,7)$ & \\
II [n $(\%)]$ & $37(43,5)$ & $40(27,2)$ & \\
III [n $(\%)]$ & $30(35,3)$ & $54(36,7)$ & \\
IV [n $(\%)]$ & $7(8,2)$ & $10(6,8)$ & \\
\hline
\end{tabular}

*: en el examen físico

**: para los estadios categorizados II y III

por medio de tamizaje, la mortalidad por cáncer de seno se reduce hasta en $35 \%$ [19-22].

De las cuarenta y dos pacientes $(85,7 \%)$ remitidas por mamografía de tamizaje anormal, en 36 se reportó el examen físico como normal; sin embargo, los especialistas encontraron hallazgos claramente positivos al examen físico. Esto constituye una situación preocupante frente a las destrezas del médico general para llevar a cabo un examen clínico de la mama en forma adecuada y resalta la importancia de generar estrategias efectivas para su enseñanza en las facultades de medicina.

Como se puede apreciar en la tabla 2, la edad promedio de las mujeres diagnosticadas por mamografía de tamizaje fue mayor en comparación con las que consultaron por sintomatología $(59,6$ versus 53,9 años $<0,05)$, dato que concuerda con lo reportado en la literatura mundial[20].

La mayoría de los casos se detectaron por sintomatología y no mediante estudios de tamizaje o detección temprana, lo cual concuerda con los estadios clínicos encontrados.

Aquellas pacientes que consultaron por sintomatología presentaron tumores más grandes $(5,1 \mathrm{~cm}$ versus $3,3 \mathrm{~cm}$, $\mathrm{p}<0,05)$, con mayor número de adenopatías al examen físico $(46,1 \%$ versus $14,3 \%, \mathrm{p}<0,05)$ y estadios clínicos más avanzados $(43,3 \%$ versus $9,5 \%, p<0,05)$ en comparación con el grupo con diag- 
nóstico por mamografía de tamizaje. No hubo diferencia estadística en cuanto al tipo de invasión histológica del tumor.

En los países desarrollados se ha demostrado que las pacientes tamizadas rutinariamente se diagnostican con mayor frecuencia en estadios I y II, y tienen mayor sobrevida, en comparación con aquellas que se tamizan irregularmente u omiten hacerlo[24-26].

En nuestra población, encontramos una importante cantidad de casos $(36,8 \%)$ en mujeres menores a la edad estipulada como la de inicio para el tamizaje de oportunidad (50 años).

En la tabla 3 se evidencia que, entre las pacientes elegibles para tamizaje de oportunidad, sólo $24,7 \%$ fueron remitidas por anomalías mamográficas, probablemente debido a un insuficiente uso de esa estrategia de detección temprana.

Se encontró, además, que existe una diferencia definitiva en el tiempo de evolución del cuadro clínico entre las mujeres mayores y menores de 50 años, que es la edad de corte para el inicio del tamizaje en Colombia. Las primeras se demoraron el doble del tiempo que les tomó a las menores de 50 años en consultar al especialista $(12,5$ meses versus 6,5 meses $<0,05)$.

Ambas situaciones se contraponen con el hecho de que este grupo de edad es el que se tamiza en nuestro país y es el objetivo principal de campañas educativas que buscan generar diagnósticos tempranos. También es llamativo que, aun cuando nuestro sistema de salud propone el tamizaje de oportunidad, la cantidad de mujeres diagnosticadas mediante esta estrategia es baja.

Otro hallazgo llamativo fue el número elevado (36\%) de casos de cáncer de seno en menores de cincuenta años, lo cual se correlaciona con los datos obtenidos en varios estudios colombianos, como el de la Clínica del Country y el del Instituto Nacional de Cancerología, en los que se reportaron $37,4 \%$ y $38 \%$ de las pacientes por debajo de esta edad, respectivamente[27, 28].

Infortunadamente, no contamos con datos que nos permitan evaluar si en Colombia ha habido un incremento en el uso de la mamografía desde la implementación del tamizaje de oportunidad. Lo cual deberá ser abordado en estudios en el futuro.

El carácter retrospectivo de nuestros datos limita la información referente a las mamografías previas, edad de inicio del tamizaje y periodicidad de la misma, que podemos obtener de las historias clínicas y, por ende, una visión más detallada de este aspecto en nuestra población. No se contó con información completa de todo el proce- 
so de tamizaje, como edad de inicio, frecuencia y número de mamografías previas. No podemos extrapolar a nivel nacional el uso del tamizaje de oportunidad en nuestra población, dadas las características de nuestros casos centro médico privado, área urbana y régimen contributivo. No obstante, consideramos nuestros datos valiosos, en la medida que no existen publicaciones que traten este aspecto a nivel nacional.

La cantidad de pacientes con carcinoma de seno detectado por mamografía es menor a la esperada. Al parecer, en nuestra población existe un limitado uso de la mamografía como herramienta de tamizaje en aquellas mujeres en quienes está indicada. Además, encontramos una demora significativa en el diagnóstico de las mujeres mayores de 50 años, objetivo de las campañas de tamizaje y concientización en Colombia.

Es necesario mejorar los tiempos de consulta y el acceso para el diagnóstico de cáncer de seno, ya que lo observado en esta cohorte d es que las pacientes se están demorando, en promedio, más de tres meses en llegar a la consulta especializada, tiempo mayor al recomendado por diferentes guías de manejo[29, 30].

La posibilidad de implementar un programa organizado de tamizaje para cáncer de seno en Bogotá, requiere que haya una reducción en los tiem- pos de demora, por parte de aseguradores y prestadores, ante la sospecha de una lesión maligna. Si no se logra reducir el tiempo de atención y tratamiento en mujeres sintomáticas, tampoco se tienen argumentos para invitar a las mujeres asintomáticas a un diagnóstico oportuno. Previamente se ha descrito que las mujeres de edad avanzada, de clase sociocultural baja o asintomáticas, tienden a consultar tardíamente, por lo que se debe brindar incentivos para garantizar un diagnóstico temprano en todos los casos[31,32].

El 36,6\% de las pacientes diagnosticadas por primera vez en el Hospital Universitario San Ignacio estaba por debajo de la edad en que se recomienda tamizar en Colombia. A pesar de que se ha demostrado que la utilidad de la mamografía como herramienta de tamizaje en mujeres entre los $40 \mathrm{y}$ 50 años de edad es controversial, y de poca utilidad en menores de 40 años por la densidad del tejido, no debemos olvidar que varios estudios colombianos han reportado proporciones de pacientes jóvenes iguales a las nuestras[27, 33, 34], lo cual debe llevar a reforzar las estrategias de detección temprana en este grupo de edad.

\section{Comentarios finales}

La mamografía de tamizaje ha cumplido su tarea de mejorar el estadio clínico en el momento del diagnóstico y reducir las tasas de mortalidad en los 
países desarrollados[35, 36]. Este estudio permite observar los resultados que brinda esta herramienta en un hospital con una población selectiva, al demostrar nuevamente que ésta logra detectar tumores en estadios más tempranos. Los hallazgos al respecto fueron de tipo exploratorio y cualitativo $\mathrm{y}$, por lo tanto, es necesario ampliar los resultados de este estudio en forma prospectiva, con el fin de evaluar el impacto del uso de la mamografía, como herramienta de tamizaje, en la supervivencia de nuestras pacientes.

\section{Bibliografía}

1. Ferlay J, Bray F, Pisani P, Parkin DM. GLOBOCAN 2000: Cancer incidence, mortality and prevalence worldwide, version 2.0. IARC Cancer Base $\mathrm{N}^{\circ} 5$. Lyon: IARC Press. 2001.

2. Schwarstsmann G. Breast cancer in South America: challenges to improve early detection and medical management of a public health problem. J Clin Oncol. 2001;19(Suppl.18):118S-24S.

3. Piñeros M, Hernández G, Bray F. Increasing mortality rate of common malignancies in Colombia: an emerging problem. Cancer. 2004;101:2285-92.

4. Vogel, VG. Breast Cancer prevention: a review of current evidence. CA Cancer J Clin. 2000; 50:156-70.

5. Shapiro S. Periodic screening for breast cancer: The HIP randomized controlled trial. Health insurance plan. J Natl Cancer Ins Monogr. 1997;22:27-30.

6. Andersson I, Janzon L. Reduced breast cancer mortality in women under age 50 : updated results from the Malmo mammographic screening program. J Natl Cancer Inst Monogr. 1997;22:63-7.

7. Tabar L, Vitak B, Chen HH, Duffy SW, Yen MF, Chiang CF, et al. the Swedish two/country trial twenty years later. Updated mortality results and new insights from long/term follow up. Radiol Clin North Am. 2000; 38:625-51.

8. Alexander FE, Anderson TJ, Brown HK, Forrest Ap, Hepburn W, Kirkpatrick AE, et al. 14 years follow up from the Edinburgh randomized trial of breast cancer screening. Lancet. 1999;353: 1903-8.

9. Miller AB, To T, Baines CJ, Wall C. Canadian Breast Screening study-2: 13year results of a randomized trial in women aged 50-59 years. J Natl Cancer Inst. 2000;92:1490-9.

10. Miller AB, To T, Baines CJ, Wall C. The Canadian Breast Screening study1 ; Breast cancer mortality after 11 to 16 years of follow up: a randomized screening trial of mammography in women age 40 to 49 years. Ann Intern Med. 2002; 137:305-15.

11. Bjurstam N, Bjorneld L, Warwick J, Sala E, Duffy SW, Nystrom L, et al. The Golthenburg Breast Screening Trial. Cancer. 2003; 97:2387-96.

12. Ballard-Barbash R, Klabunde C, Paci E, Broeders M, Coleman EA, Fracheboud J, Bouchard F, Rennert G, Linos A, Shapiro S. International Breast Cancer Screening Network (IBSN). Breast cancer screening in 21 countries: delivery of services, notification of results, and outcomes ascertainment. Eur J Cancer Prev. 1999; 8:417-26.

13. Anderson Bo, Braun S, Lim S, Smith RA, Taplin S, Thomas DB, et al. Early detection of breast cancer in countries 
with limited resources. Breast $J$. 2003;9(Suppl.2):S51-9.

14. República de Colombia. Ministerio de la Protección Social, Instituto Nacional de Cancerología. Recomendaciones para la detección temprana del cáncer de mama en Colombia. Bogotá: Instituto Nacional de Cancerología. 2007.

15. Suter LG, Nakano CJ, Elmore JG. The personal costs and convenience of screening mammography. J Womens Health Gend Based Med. 2002;11: 667-72.

16. Pearlman DN, Rakowski W, Ehrich B. Mammography, clinical breast exam, and Pap testing: correlates of combined screening. Am J Prev Med. 1996;12:57-64,.

17. República de Colombia. Ministerio de Salud. Resolución 412, 25 de febrero de 2000, por la cual se establecen las actividades, procedimientos e intervenciones de demanda inducida y obligatorio cumplimiento y se adoptan las normas técnicas y guías de atención para el desarrollo de las acciones de protección específica, detección temprana y la atención de enfermedades de interés en salud pública. Bogotá: Imprenta Nacional. 2000.

18. Lannin DR, Mathews HF, Miychell J, Swanson MS, Swanson FH, Edwards MS. Influence of socioeconomic and cultural factors on racial differences in late-stage presentation of breast cancer. JAMA. 1998;279:1801-7.

19. Whitman G, Sheppard D, Phelps M. Breast cancer staging. Semin Roentgenol. 2006;41:91-104.

20. Barth RJ, Gibson GR, Carney PA, Mott LA, Becher RD, Poplack SP. Detection of breast cancer on screening mammography allows patients to be treated with less-toxic therapy. AJR. 2005;184:324-9.

21. Veronesi U, Boyle P, Goldhirsch A, Orecchia R, Viale G. Breast cancer. Lancet. 2005;365:1727-41.

22. Jonsson H, Nyström L, Törnberg S, Lundgren B, Lenner P. Service screening with mammography. Long term effects on breast cancer mortality in the county of Gävleborg, Sweden. Breast. 2003;12:183-93.

23. Seltzer MH. Breast complaints, biopsies, and cancer correlated with age in 10,000 consecutive new surgical referrals. Breast J. 2004;10:111-7.

24. Spencer DB, Potter JE, Chung MA, Fulton J, Hebert W, Cady B. Mammographic screening and disease presentation of breast cancer patients who die of disease. Breast J. 2004;10:298-303.

25. Gill PG, Farshid G, Luke CG, Roder DM. Detection by screening mammography is a powerful independent predictor of survival in women diagnosed with breast cancer. Breast. 2004;13:15-22.

26. Buitatti E, Barchielli A, Bartolacci S, Federico M, De Lisi V, Bucchi L, et al. The impact of organized screening programmes on the stage-specific incidence of breast cancer in some Italian areas. Eur J Cancer. 2003;39:1776-82.

27. Robledo JF, Caicedo JJ, Deantonio R. Análisis de sobrevida en una cohorte de 1328 pacientes con carcinoma de seno. Rev Colomb Cir. 2005; 20:4-20.

28. Piñeros M, Sánchez R, Cendales R, Perry F, Ocampo R, García OA. Características sociodemográficas, clínica y de la atención de mujeres con cáncer de mama en Bogotá. Rev Colomb Cancerol. 2008; 12:181-90. 
29. Canadian Society for Surgical Oncology position statement. Fecha de consulta: 13 de marzo de 2009. Disponible en: http://www.cos.ca/csso.

30. Robinson D, Bell CM, Møller H, Basnet I, Effect of the UK government's 2week target on waiting times in women with breast cancer in southeast England. Br J Cancer. 2003;89:492-6.

31. Ramírez AJ, Westcombe AM, Burgess CC, Sutton S, Littlejohns P, Richards MA. Factors predicting delayed presentation of symptomatic breast cancer: a systematic review. Lancet. 1999;353: 1127-31.

32. Burgess CC, Ramírez AJ, Richards MA, Love SB. Who and what influences delayed presentation in breast cancer? Br J Cancer. 1998;77:1343-8.
33. González-Mariño MA. Cáncer de seno en la Clínica San Pedro Claver de Bogotá, 2004. Rev Salud Pública. 2006;8:163-9.

34. Pardo C, Murillo R. Casos nuevos de cáncer en el Instituto Nacional de Cancerología, Colombia. Rev Col de Cancerol. 2002;7:4-19.

35. Peto R, Boreham J, Clarke M, Davies $\mathrm{C}$, Beral V. UK and USA breast cancer deaths down $25 \%$ in year 2000 at ages 20-69 years. Lancet. 2000;355:1822.

36. Hermon C, Beral V. Breast cancer mortality rates are leveling off or beginning to decline in many western countries: analysis of time trends, agecohort and age-period models of breast cancer mortality in 20 countries. $\mathrm{Br} \mathrm{J}$ Cancer. 1996;73:955-60. 